\title{
IIR Multiple Notch Filter Design Based on Allpass Filter
}

\author{
Soo-Chang Pei ${ }^{*}$ and Chien-Cheng Tseng \\ Department of Electrical Engineering \\ National Taiwan University \\ Taipei, Taiwan, R.O.C.
}

\begin{abstract}
In this paper, real coefficient IIR notch filter design problem is investigated. The specification of notch filter is first transformed into that of allpass filter. Then we develop an effective approach to design this desired allpass filter. The realization of proposed notch filter is equivalent to the realization of an allpass filter. Due to the mirror-image symmetry relation between the numerator and denominator polynomials of allpass filter, the notch filter can be realized by a computationally efficient lattice structure with very low sensitivity. Moreover, some design and application examples are presented to examine the effectiveness of proposed method.
\end{abstract}

\section{Introduction}

In many applications of signal processing it is desired to remove narrowband or sinusoidal interferences while leaving the broadband signal unchanged. Examples are in the areas of communication, control, biomedical engineering and others [1]-[12]. A typical one is to cancel $60 \mathrm{~Hz}$ power line interference in the recording of electrocardiogram (ECG) [1][2]. Usually this task can be achieved by the notch filters characterized by a unit gain at all frequencies except at

*Author for correspondence the sinusoidal frequencies in which their gain is zero. So far, several methods have been developed to design IIR and FIR notch filters very efficiently [3]- [12]. When the frequencies of narrowband interferences are known in advance, fixed notch filters can be used [3][6]. However, when sine wave frequencies are unknown or time-varying, adaptive notch filters are applicable [7]-[12]. In this paper, we will focus on fixed IIR notch filter design problem.

The IIR notch filter $H(z)$ studied here has the following form:

$$
H(z)=\frac{1}{2}(1+A(z))
$$

where $A(z)$ is an allpass filter. Compared with conventional methods, the main advantage of this form is that the mirror-image symmetry relation between the numerator and denominator polynomials of $A(z)$ can be exploited to obtain a computationally efficient lattice filter realization with very low sensitivity. When the $A(z)$ is chosen as second order real-valued coefficient allpass filter, the design procedures and a catalogue of minimum-multiplier structures are detailed in [4]. However, the design problem of high order notch filter based on this form have not been investigated until now. The purpose of this paper is to develop an effective approach to solve this design problem.

The paper is organized as follows. In section II, a new method for real coefficient notch filter design will 
be developed. First, we transform the specification of notch filter into that of allpass filter. As a result, high order notch filter design problem becomes an allpass filter design problem. Next, a simple design procedure of allpass filter is developed and an efficient lattice realization form is also studied. Finally, a design example is presented and one experiment of the power line interference removal in ECG signal is shown.

\section{Real Coefficient Notch Fil- ter Design}

In this section, real coefficient notch filter design based on IIR allpass filter will be investigated. Generally, the input of real coefficient notch filter has the following form:

$$
\begin{aligned}
x(n) & =s(n)+\sum_{k=1}^{M} A_{k} \sin \left(n \omega_{N k}+\phi_{k}\right) \\
& =s(n)+d(n)
\end{aligned}
$$

where $s(n)$ is desired signal, $d(n)$ is sinusoidal interference and frequencies $\omega_{N k} \in(0, \pi)$ for $k=1, \cdots, M$. In order to extract $s(n)$ from corrupted signal $x(n)$ undistortedly, the specification of ideal notch filter is given by

$$
H\left(e^{j \omega}\right)= \begin{cases}0 & \omega=\omega_{N k}, k=1 \cdots M \\ 1 & \text { otherwise }\end{cases}
$$

So far, many methods have been developed to design IIR and FIR notch filter, such as bilinear transform and Lagrange multiplier method [3]-[6]. Here, a new method is developed as follows:

2.1 Specification transformation:

In the following, we will use the relation $H(z)=$ $\frac{1}{2}(1+A(z))$ to transform the specification of high order notch filter into that of allpass filter. As a result, the notch filter design problem becomes an allpass filter design problem

The transfer function of a $2 M$-order real coefficient allpass filter $A(z)$ is defined by

$$
A(z)=\frac{a_{2 M}+\cdots+a_{1} z^{-2 M+1}+z^{-2 M}}{1+a_{1} z^{-1}+\cdots+a_{2 M} z^{-2 M}}
$$

Since magnitude response of $A(z)$ is equal to unity for all frequency, the frequency response can be written as

$$
A\left(e^{j \omega}\right)=e^{j \theta_{A}(\omega)}
$$

where $\theta_{A}(\omega)$ is the phase response. From eq(1), we have the frequency response of notch filter $H(z)$ as follows:

$$
H\left(e^{j \omega}\right)=\frac{1}{2}\left(1+e^{j \theta_{A}(\omega)}\right)
$$

The $\theta_{A}(\omega)$ of a stable allpass filter is zero when $\omega=0$, $-2 M \pi$ when $\omega=\pi$, and is required to decrease monotonically with increasing frequency [13]. Thus, when $\omega$ goes from 0 to $\pi$ radians, the phase $\theta_{A}(\omega)$ goes from 0 to $-2 M \pi$. Based on this property, we have the fol lowing observations:

(1) There exists $M$ frequency points $\omega_{1}<\omega_{2}<\cdots<$ $\omega_{M}$ such that $\theta_{A}\left(\omega_{n}\right)=-(2 n-1) \pi$, that is,$H\left(e^{j \omega_{n}}\right)=$ 0 for $n=1 \cdots M$.

(2) There exists $M$ frequency points $\bar{\omega}_{1}<\bar{\omega}_{2}<\cdots<$ $\bar{\omega}_{M}$ such that $\theta_{A}\left(\bar{\omega}_{n}\right)=-(2 n-1) \pi+\frac{\pi}{2}$, that is, $\left|H\left(e^{j \bar{\omega}_{n}}\right)\right|=\left|\frac{1}{2}(1-j)\right|=\frac{1}{\sqrt{2}}$ for $n=1 \cdots M$.

(3) There exists $M$ frequency points $\hat{\omega}_{1}<\hat{\omega}_{2}<\cdots<$ $\hat{\omega}_{M}$ such that $\theta_{A}\left(\hat{\omega}_{n}\right)=-(2 n-1) \pi-\frac{\pi}{2}$, that is , $\left|H\left(e^{j \hat{\omega}_{n}}\right)\right|=\left|\frac{1}{2}(1+j)\right|=\frac{1}{\sqrt{2}}$ for $n=1 \cdots M$.

(4) There exists $M+1$ frequency points $0=\tilde{\omega}_{0}<$ $\tilde{\omega}_{1}<\cdots<\tilde{\omega}_{M}=\pi$ such that $\theta_{A}\left(\tilde{\omega}_{n}\right)=-2 n \pi$, that is ,$H\left(e^{j \bar{\omega}_{n}}\right)=1$ for $n=0,1 \cdots M$.

When $M=2$, a graphic interpretation of these four observations is shown in Fig. 1. It is obvious that four statements are valid intuitively. Moreover, we can show that $\left|H\left(e^{j \omega}\right)\right|=\left|\cos \left(\frac{\theta_{A}(\omega)}{2}\right)\right|$, so the maximum gain of magnitude response of notch filter is unity. Based on the above observations, if we want to design a notch filter $H(z)$ which satisfies the specification shown in Fig.2, we only need to make the following assignments of the phase $\theta_{A}(\omega)$ of allpass filter $A(z)$ :

(1) $\theta_{A}\left(\omega_{N n}\right)=-(2 n-1) \pi$.

(2) $\theta_{A}\left(\omega_{N n}-\frac{B W_{n}}{2}\right)=-(2 n-1) \pi+\frac{\pi}{2}$.

(3) $\theta_{A}\left(\omega_{N n}+\frac{B W_{n}}{2}\right)=-(2 n-1) \pi-\frac{\pi}{2}$.

where $n=1 \cdots M$ and the notch frequency ponits $\omega_{N n}$ satisfy $\omega_{N 1}<\cdots<\omega_{N M}$. In most practical applications, the $3 \mathrm{~dB}$ rejection $B W_{n}$ will be very small. Thus, we have the following fact: 
Fact: If $B W_{n}$ is very small, $\theta_{A}\left(\omega_{N n}\right)=-(2 n-1) \pi$ and $\theta_{A}\left(\omega_{N n}-\frac{B W_{n}}{2}\right)=-(2 n-1) \pi+\frac{\pi}{2}$, then it can be shown that

$$
\theta_{A}\left(\omega_{N n}+\frac{B W_{n}}{2}\right) \approx-(2 n-1) \pi-\frac{\pi}{2}
$$

Pf: Applying the Taylor series expansion, we obtain

$$
\theta_{A}\left(\omega_{N n}-\frac{B W_{n}}{2}\right) \approx \theta_{A}\left(\omega_{N n}\right)-\theta_{A}^{\prime}\left(\omega_{N n}\right) \frac{B W_{n}}{2}
$$

(8) In the following, we first develop an approach to dewhere $\theta_{A}^{\prime}(\omega)$ denotes the derivative of $\theta_{A}(\omega)$ with respect to $\omega$. Based on the assumption $\theta_{A}\left(\omega_{N n}\right)=$ $-(2 n-1) \pi$ and $\theta_{A}\left(\omega_{N n}-\frac{B W_{n}}{2}\right)=-(2 n-1) \pi+\frac{\pi}{2}$, eq(8) becomes

$$
\theta_{A}^{\prime}\left(\omega_{N n}\right) \frac{B W_{n}}{2} \approx-\frac{\pi}{2}
$$

Using Taylor series expansion and eq(9), we have

$$
\begin{aligned}
\theta_{A}\left(\omega_{N n}+\frac{B W_{n}}{2}\right) & \approx \theta_{A}\left(\omega_{N n}\right)+\theta_{A}^{\prime}\left(\omega_{N n}\right) \frac{B W_{n}}{2} \\
& \approx-(2 n-1) \pi-\frac{\pi}{2}
\end{aligned}
$$

The proof is completed.

This fact tells us that assignments (1) and (2) imply assignment (3) if rejection bandwidth $B W_{n}$ is very small. Thus, three assignments can be reduced to two assignments as follows:

(1) $\theta_{A}\left(\omega_{N n}\right)=-(2 n-1) \pi$.

(2) $\theta_{A}\left(\omega_{N n}-\frac{B W_{n}}{2}\right)=-(2 n-1) \pi+\frac{\pi}{2}$.

where $n=1 \cdots M$ and the notch frequency ponits $\omega_{N n}$ satisfy $\omega_{N 1}<\cdots<\omega_{N M}$. After suitable arrangement, these two assigments are equivalent to the following condition in $2 M$ frequency sampling points. When the frequency point

$$
w_{n}=\omega_{N\left\lfloor\frac{n+1}{2}\right\rfloor}-\frac{1}{2}\left(1-(-1)^{\bmod (n, 2))} \frac{B W_{\left\lfloor\frac{n+1}{2}\right\rfloor}}{2}\right.
$$

, the desired phase response is specified by

$$
\begin{aligned}
\theta_{A}\left(\omega_{n}\right) & =-\left(2\left\lfloor\frac{n+1}{2}\right\rfloor-1\right) \pi \\
& +\frac{1}{2}(1-(-1) \bmod (n, 2)) \frac{\pi}{2}
\end{aligned}
$$

where $n=1 \cdots 2 M,\lfloor x\rfloor$ denotes the largest integer which is smaller than or equal to $x$, and $\bmod (x, 2)$ denotes the remainder when $x$ is divided by 2 .
So far, the specification of notch filter in Fig.2 has been transformed into the specification of allpass filter. Thus, we only need to design an allpass filter $A(z)$ which satisfies these $2 M$ requirements in eq(12), then $\frac{1}{2}(1+A(z))$ is the desired notch filter.

2.2 Design Procedure and Lattice Form Realization sign allpass filter $A(z)$ such that the phase response $\theta_{A}(\omega)$ satisfies the $2 M$ requirements in eq(12) exactly. Then, an efficient lattice-form realization is studied.

\subsection{1: Design Procedure:}

It is easy to show that the phase response $\theta_{A}(\omega)$ of $A(z)$ in eq(4) can be written as

$$
\theta_{A}(\omega)=-2 M \omega+2 \arctan \left(\frac{\sum_{k=1}^{2 M} a_{k} \sin (k \omega)}{1+\sum_{k=1}^{2 M} a_{k} \cos (k \omega)}\right)
$$

From eq(12), we see that the phase response $\theta_{A}(\omega)$ is given in $2 M$ points $\omega_{n}, n=1 \cdots 2 M$. Thus, we obtain a sets of equations

$$
\frac{\sum_{k=1}^{2 M} a_{k} \sin \left(k \omega_{n}\right)}{1+\sum_{k=1}^{2 M} a_{k} \cos \left(k \omega_{n}\right)}=\tan \left(\beta_{n}\right) \quad n=1,2, \cdots, 2 M
$$

where $\beta_{n}=\frac{1}{2}\left[\theta_{A}\left(\omega_{n}\right)+2 M \omega_{n}\right]$. After some manipulations, this expression can be rewritten as

$$
\begin{gathered}
\sum_{k=1}^{2 M}\left[\sin \left(k \omega_{n}\right)-\tan \left(\beta_{n}\right) \cos \left(k \omega_{n}\right)\right] a_{k}=\tan \left(\beta_{n}\right) \\
n=1, \cdots, 2 M
\end{gathered}
$$

which is linear equation of filter coefficients $a_{k}$. Thus, it can be expressed in the following matrix form

$$
\mathbf{Q} \mathbf{a}=\mathbf{p}
$$

where two vectors are

$$
\begin{aligned}
& \mathbf{a}=\left[\begin{array}{ll}
a_{1} & a_{2} \cdots a_{2 M}
\end{array}\right]^{t} \\
& \mathbf{p}=\left[\begin{array}{ll}
\tan \left(\beta_{1}\right) & \tan \left(\beta_{2}\right) \cdots \tan \left(\beta_{2 M}\right)
\end{array}\right]^{t}
\end{aligned}
$$

and the elements of the matrix $Q$ are given by

$$
\begin{gathered}
q_{n k}=\sin \left(k \omega_{n}\right)-\tan \left(\beta_{n}\right) \cos \left(k \omega_{n}\right) \quad n=1, \cdots, 2 M \\
k=1, \cdots, 2 M
\end{gathered}
$$


By solving linear simultaneous equations (16), the desired solution is given by

$$
\mathbf{a}=\mathbf{Q}^{-1} \mathbf{p}
$$

Finally, we summarize the entire design procedure of real coefficient notch filter as follows:

(1) Prescribe notch frequencies $\omega_{N 1}<\omega_{N 2}<\cdots<$ $\omega_{N M}$ and $3 \mathrm{~d} B$ rejection bandwidth $B W_{1}, B W_{2}, \cdots, B W_{M}$.

(2) Use eq(11)(12) to compute $w_{n}$ and $\theta_{A}\left(\omega_{n}\right), n=$ $1,2, \cdots 2 M$

(3) Use eq(17)(18) to calculate $Q$ and $p$. Then, find the solution $\mathbf{a}=\mathrm{Q}^{-1} \mathbf{p}$.

(4) The notch filter is obtained as

$$
H(z)=\frac{1}{2}\left(1+\frac{a_{2 M}+\cdots+a_{1} z^{-2 M+1}+z^{-2 M}}{1+a_{1} z^{-1}+\cdots+a_{2 M} z^{-2 M}}\right)
$$

\subsection{2: Lattice Form Realization:}

Since $H(z)=\frac{1}{2}(1+A(z))$, the notch filter can be implemented using the structure shown in Fig.3(a). Thus, the notch filter realization is equivalent to the realization of allpass filter. Due to the mirror-image symmetry relation between the numerator and denominator polynomials of allpass filter, the $A(z)$ can be realized by computationally efficient lattice structure shown in Fig.3(b). This structure has minimum multipliers and delays. When filter coefficients $a_{i}$ of direct-form allpass filter are obtained in term of proposed design procedure, the lattice coefficients $k_{i}, i=$ $1, \cdots, 2 M$ can be obtained by using the transform method described in [14]. Moreover, the frequency response of notch filter is very insensitive with respect to the coefficients $k_{i}, i=1, \cdots, 2 M$, and notch filter is stable if $\left|k_{i}\right|<1, i=1, \cdots, 2 M$.

\subsection{Design Example and Application:}

In the following, a design example and an experiment of the power line interference removal in ECG signal are presented. Both examples are performed with the MATLAB language in an IBM PC compatible computer.

Example 1: Sixth order notch filter design:

In this example, the specification of a sixth order notch filter is chosen as

$$
\omega_{1 N}=0.1 \pi \quad B W_{1}=0.005 \pi
$$

$$
\begin{array}{cc}
\omega_{2 N}=0.2 \pi & B W_{2}=0.005 \pi \\
\omega_{3 N}=0.6 \pi & B W_{3}=0.01 \pi
\end{array}
$$

Using the proposed design method, we obtain the filter coefficients of a sixth order allpass filter as follows:

$$
\begin{array}{llrl}
a_{1} & =-2.8678 & a_{4} & =3.5463 \\
a_{2} & =3.7868 & a_{5} & =-2.5861 \\
a_{3} & =-3.6666 & a_{6} & =0.8793
\end{array}
$$

The phase response $\theta_{A}(\omega)$ and magnitude response of notch filter are shown in Fig.4. From this result, we see that the specification is well satisfied. Moreover, we use the transform method developed in [14] to compute the lattice filter coefficients $k_{i}$ as follows:

$$
\begin{array}{ll}
k_{1}=-0.9158 & k_{4}=0.2295 \\
k_{2}=0.9424 & k_{5}=-0.2841 \\
k_{3}=-0.6604 & k_{6}=0.8793
\end{array}
$$

It is clear that the $\left|k_{i}\right|<1$ for $i=1, \cdots 6$. Thus, the notch filter is a stable filter.

Exapmle 2: Power line interference canceling in ECG signal:

A major problem in the recording of ECG is that the measurement signals degraded by the power line interference. One source of interference is electrical fileld characterized by noise concentrated at the fundamental frequency $60 \mathrm{~Hz}$. The other source is magnetic field which is characterized by high harmonic content. The harmonics are due to the nonlinear characteristics of transformer cores in the power supply [2]. Thus, use high order notch filter to reduce interference becomes an important subject in ECG measurement.

In this example, we will use the proposed notch filter to remove power line interference. The samples used here have 8 bits and the sampling rate is 800 $\mathrm{Hz}$. Fig.5(a) shows the input waveform which is ECG signal corrupted by harmonic interference with frequencies $60 \mathrm{~Hz}, 180 \mathrm{~Hz}, 300 \mathrm{~Hz}$. The specification of notch filter is chosen as

$$
\begin{array}{ll}
\omega_{1 N}=0.15 \pi & B W_{1}=0.005 \pi \\
\omega_{2 N}=0.45 \pi & B W_{2}=0.005 \pi \\
\omega_{3 N}=0.75 \pi & B W_{3}=0.005 \pi
\end{array}
$$


Fig.5(b) shows the waveform of notch filter output with zero initial. From this result, it is obvious the interference has been removed by our high order notch filter except some transient states appear at the beginning.

\section{Conclusion}

This paper presents a new method to design real coefficient notch filters for a prescribed notch frequencies and $3 \mathrm{~dB}$ rejection bandwidths. This notch filter can be realized by a computationally efficient lattice structure with very low sensitivity. Some design and application examples have been demonstrated it$s$ good performance. However, only one dimensional (1D) filter design problems are investigated. Thus, it is interesting to extend this method to $2 \mathrm{D}$ notch filter designs. This topic will be studied in the future.

\section{References}

[1] J.C.Huhta and J.G.Webster, "60-Hz interference in Electrocadiograph", IEEE Trans. Bio-Med. Eng., Vol. BEM-20, pp.91-101, March 1973.

[2] C.D.McManus, D.Neubert, and E.Cramer, "Characterization and elimination of $\mathrm{AC}$ noise in electrocardiograms: a comparison of digital filtering methods", Computers and Biomedical research, pp. 48-67, 1993.

[3] R.Carney, "Design of a digital notch filter with tracking requirements", IEEE Trans. Space Electron. Telem. Vol. SET-9, pp.109-114, Dec.1963.

[4] K.Hirano, S.Nishimura, and S.K.Mitra, "Design of digital notch filters", IEEE Trans. Circuits and Syst., Vol. CAS-21, pp. 540-546, July 1974.

[5] M.H.Er, "Designing notch filter with controlled null width", Signal Processing, Vol.24, No.3, pp.319-329, Sep. 1991

[6] G.W.Medlin, "A novel design technique for tuneable notch filters", Int'l symposium on Circuits and Systems, New Orleans, Louisiana, U.S.A., pp.471-474, May 1990.

[7] D.V.B.Rao and S.Y.Kung, "Adaptive notch filtering for the retrieval of sinusoids in noise", IEEE Trans. Acoust. Speech, Signal processing, Vol. ASSP-32 pp.791-802 Aug. 1984

[8] A.Nehorai, "A minimal parameter adaptive notch filter with constrained poles and zeros", IEEE Trans. Acoust. Speech, Signal processing, Vol. ASSP-33 pp.983-996 Aug. 1985

[9] T.S.Ng, "Some aspects of an adaptive digital notch filter with constrained poles and zeros", IEEE Trans. Acoust. Speech, Signal processing, Vol. ASSP-35 pp.158-161, Feb. 1987

[10] J.M.Travassos-Romano and M.Bellanger, "Fast least squares adaptive notch filtering", IEEE Trans. Acoust. Speech, Signal processing, Vol. ASSP-36 pp.1536-1540 Sep. 1988

[11] J.F.Chicharo and T.S.Ng, "Gradient-based adaptive IIR notch filtering for frequency estimation", IEEE Trans. Acoust. Speech, Signal processing, Vol. ASSP-38 pp.769-777, May 1990

[12] J.A.Chambers and A.G.Constantinides, "Frequency tracking using constrained adaptive notch filters synthesised from allpass sections", Proc. Inst. Elect. Eng., Vol.137, pt.F, No.6, pp.475-481, Dec. 1990

[13] X.Zhang and H.Iwakura, "Novel method for designing digital allpass filters based on eigenvalue problem", Electronics Letters, Vol.29, No.14, pp.1279-1281, July 1993

[14] A.H.Gray,Jr. and J.D.Markel, "Digital lattice and ladder filter synthesis", IEEE Trans. Audio Electroacoust., Vol. AU-21, No.6, pp.491-500, Dec. 1973 

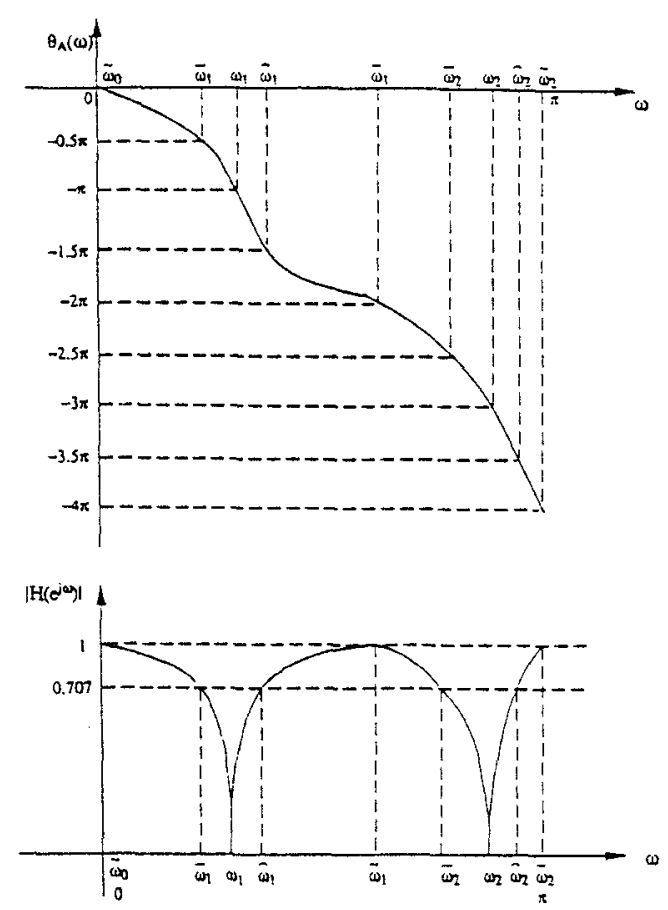

Fig. 1 Graphic inerpreation of four observacions (a) phase response of allpass filter (b) magaioude response of notch filter.

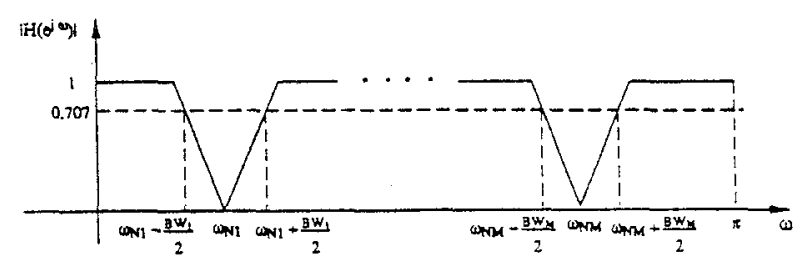

Fig.2 The prescribed specification of noteh silter.

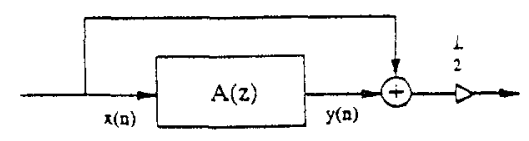

(a)

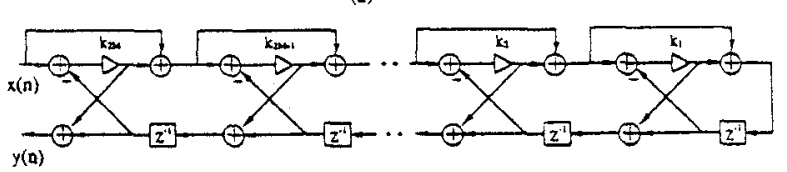

(b)

Fig.3 (a) The realizasion of notch filter. (b) The latrice form realizacion of allpass Elter.

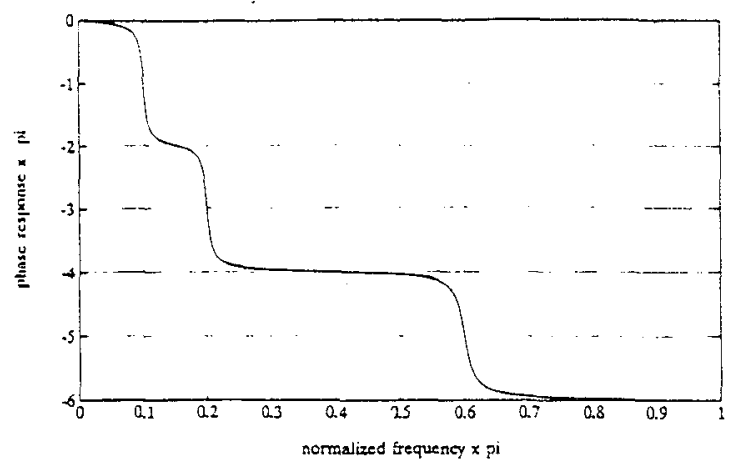

(a)

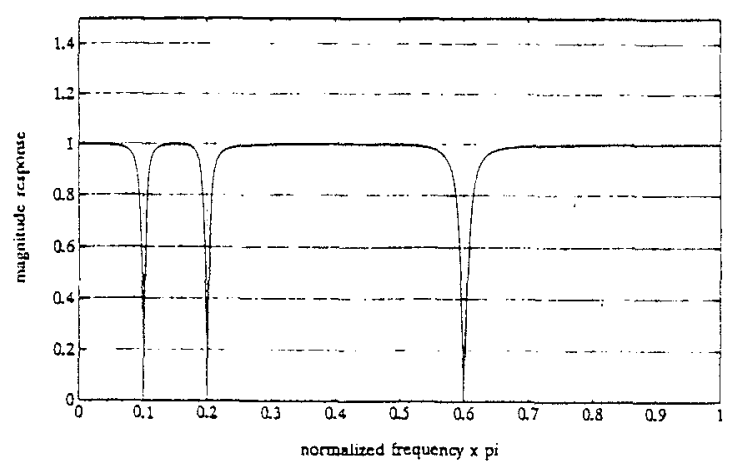

(b)

Fig.4 The results of example : (a) the phase response of allpass filter (b) the magnitude response of notch filter.
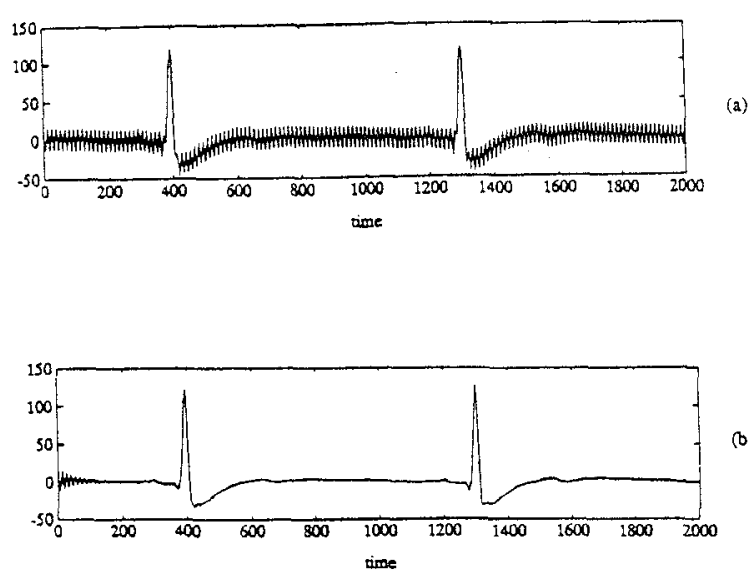

Fig-5 Power line interference canceling in ECG signal (a) the waveform of noteh filter inpus (b) the waveform of nocch fileer ouput 